\title{
Impacto da complexidade da farmacoterapia sobre parâmetros bioquímicos e pressóricos no diabetes mellitus
}

\author{
Impact of the complexity of pharmacotherapy on biochemical and \\ pressure parameters in diabetes mellitus
}

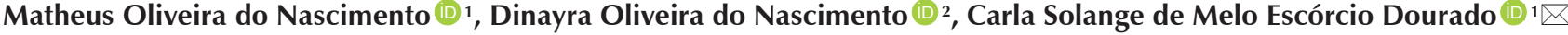 \\ 1 Universidade Federal do Piauí, Centro de Ciências da Saúde, Coordenação do Curso de Farmácia. Teresina, PI, Brasil. \\ 2 Prefeitura de Timon, Secretaria Municipal de Saúde. Timon, MA, Brasil.
}

Como citar este artigo (How to cite this article):

Nascimento MO, Nascimento DO, Dourado CSME. Impacto da complexidade da farmacoterapia sobre parâmetros bioquímicos e pressóricos no diabetes mellitus (Impact of the complexity of pharmacotherapy on biochemical and pressure parameters in diabetes mellitus). Sci Med. 2019;29(2):e33175. https://doi.org/10.15448/1980-6108.2019.2.33175

\section{RESUMO}

OBJETIVOS: Avaliar a complexidade da farmacoterapia no diabetes melito numa unidade básica de saúde e investigar eventuais correlações com variáveis bioquímicas e pressóricas.

MÉTODOS: Trata-se de um estudo observacional, descritivo com delineamento transversal, que incluiu todos os indivíduos com diagnóstico primário de diabetes tipo 2 e com prescrição preenchida com pelo menos um antidiabético oral. A quantificação da complexidade do regime terapêutico foi realizada conforme o índice da complexidade da farmacoterapia, e este foi correlacionado com variáveis do perfil bioquímico: glicemia capilar, creatinina e filtração glomerular, e pressórico: pressão arterial sistólica e diastólica, através da análise de correlação de Spearman.

RESULTADOS: A média do índice de complexidade da farmacoterapia foi de $9,42 \pm 0,48$ pontos, sendo mais proeminente na seção B $(5,03 \pm 2,57)$. O índice esteve correlacionado com a glicemia capilar $(r h o=0,538)$, pressão arterial sistólica $($ rho $=0,520)$, creatinina $($ rho $=-0,406)$ e filtração glomerular $(\mathrm{rho}=0,566)$. Esta correlação foi diretamente proporcional ao aumento da glicemia capilar $(\mathrm{p}<0,01)$, pressão arterial sistólica $(\mathrm{p}<0,01)$ e filtração glomerular $(\mathrm{p}<0,01)$, e inversamente proporcional à creatinina $(\mathrm{p}<0,05)$.

CONCLUSÕES: Nossos achados revelaram que a polifarmácia no diabete melito contribuiu para a geração da complexidade da farmacoterapia, resultando em não adesão ao tratamento prescrito e consequentemente contribuindo, principalmente, para o descontrole glicêmico e pressórico.

DESCRITORES: Atenção primária à saúde; tratamento farmacológico; glicemia.

\section{ABSTRACT}

AIMS: Assess the complexity of pharmacotherapy in diabetes mellitus in a basic health unit and investigate possible correlations with biochemical and pressure variables.

METHODS: It is study observational, descriptive, cross-sectional study that included all patients with a primary diagnosis of type 2 diabetes and a prescription filled with at least one oral antidiabetic agent. The quantification of the complexity of the therapeutic regimen was performed according to the complexity index of the pharmacotherapy; and it was correlated with variables of the biochemical profile: capillary glycemia, creatinine and glomerular filtration, and pressure systolic and diastolic blood pressure, using the Spearman correlation analysis.

RESULTS: The median of the complexity index of pharmacotherapy was $9.42 \pm 0,48$ points, being more prominent in section B (5.03 \pm 2.57 ). The index was correlated with capillary glycemia $(\mathrm{rho}=0.538)$, systolic blood pressure $(\mathrm{rho}=0.520)$, creatinine $($ rho $=-0.406)$ and glomerular filtration $(\mathrm{rho}=0.566)$. This correlation was directly proportional to the increase in capillary glycemia $(\mathrm{p}<0.01)$, systolic blood pressure $(\mathrm{p}<0.01)$ and glomerular filtration $(\mathrm{p}<0,01)$, and inversely proportional to creatinine $(\mathrm{p}<0.05)$.

CONCLUSIONS: Our findings revealed that polypharmacy in diabetes mellitus contributed to the generation of pharmacotherapy complexity, resulting in non-adherence to the prescribed treatment and consequently contributing mainly to the lack of glycemic and pressure control.

KEYWORDS: Primary health care; drug therapy; glycemia. 
Abreviaturas: DM, diabete melito; DM2, diabete melito tipo 2; UBS, unidade básica de saúde; MRCI, Medication Regimen Complexity Index; ICFT, Índice da Complexidade da Farmacoterapia; PAS, pressão arterial sistólica; PAD, pressão arterial diastólica.

\section{INTRODUÇÃO}

O diabete melito (DM) é considerado como uma síndrome heterogênea de distúrbios metabólicos, decorrente de defeitos na secreção de insulina, na sua ação, ou em ambos. Com alta prevalência e natureza crônica, as complicações da doença são caracterizadas por alterações vasculares e neuropáticas, apresentando, como principal efeito, a hiperglicemia [1].

Impedir o início do DM (prevenção primária) ou prevenir o estabelecimento das complicações crônicas e agudas (prevenção secundária) é extremamente fundamental, visto que as complicações micro e macrovasculares estão fortemente associadas ao aumento da mortalidade. Para isso, modificações do estilo de vida, complementada com farmacoterapia e monitorização frequente da glicemia, torna-se necessário para retardar a progressão do diabetes e evitar complicações crônicas irreversíveis [2].

Os antidiabéticos orais promovem, com controle rigoroso do paciente, diminuição da incidência de complicações agudas e crônicas, além de possuírem boa aceitação e pouca influência no aumento do peso em comparação à insulina. Portanto, estes fármacos devem ser os principais agentes de primeira escolha para o tratamento do DM tipo 2 (DM2) não responsivo a medidas não farmacológicas isoladas [3].

Todavia, a maioria dos indivíduos com DM2 apresentam, concomitantemente, outras doenças, tais como obesidade, hipertensão arterial e dislipidemia, o que torna o seu manejo bastante complexo, requerendo o uso concomitante de múltiplos fármacos, bem como a obrigatoriedade de usar em seu dia-a-dia uma série de novas tecnologias para obter o controle glicêmico $[1,4]$. Assim, esses regimes podem ser desafiadores para implementar e sustentar a longo prazo o controle metabólico da doença, contribuindo para a não adesão ao tratamento [5].

A complexidade do regime medicamentoso é uma realidade vivenciada por indivíduos com doenças crônicas, visto que o seu aumento está diretamente associado à não-adesão da farmacoterapia e à erros de medicação. Uma revisão sobre a adesão ao tratamento no DM2 demonstrou que muitos diabéticos tomaram menos do que a quantidade prescrita de antidiabéticos orais, provocando uma redução das taxas gerais de adesão ao longo do tempo [6].

Neste contexto, diferentes métodos já foram utilizados para quantificar a complexidade dos regimes medicamentosos. O Medication Regimen Complexity Index é a ferramenta mais utilizada, pois quantifica a complexidade considerando não somente o número de medicamentos, como também estabelece uma pontuação ponderada para os tipos de formas de dosagem prescritas, frequências da dosagem e estratégias de administração adicionais das medicações [7]. Portanto, vale ressaltar que a complexidade clínica inerente ao tratamento do DM necessita de uma assistência interdisciplinar envolvendo o apoio contínuo dos serviços de profissionais da saúde, tornando o paciente coparticipante nas tomadas de decisões [8].

Diante do exposto e, considerando a relevância do tema, o objetivo deste estudo foi investigar a influência da complexidade da farmacoterapia sobre parâmetros laboratoriais e pressóricos no DM2 usuários de uma unidade básica de saúde (UBS).

\section{MÉTODOS}

Trata-se de um estudo observacional, descritivo com delineamento transversal e analítico, realizado em uma unidade básica de saúde do bairro Parque Alvorada, localizada no município de Timon-MA, em março de 2017. A UBS situa-se em uma região carente da cidade e oferecia consultas médicas, odontológicas e de enfermagem com atendimentos a hipertensos e diabéticos, bem como pré-natal e puericultura. Este estudo foi aprovado pelo Comitê de ética em pesquisa humana da Universidade Federal do Piauí, sob o CAAE $\mathrm{n}$ - 63887916.3.0000.5214, parecer $\mathrm{n}$ - 1.895.406.

A população deste estudo incluiu todos os indivíduos atendidos na UBS com diagnóstico primário de DM2 e com prescrição preenchida com pelo menos um antidiabético oral. A data da primeira prescrição foi considerada a data de cadastro do paciente na UBS. Foi incluído no estudo indivíduos que utilizavam hipoglicemiantes orais ou insulina, de ambos os gêneros, sendo eliminados apenas aqueles os quais não possuíam registros dos medicamentos prescritos. O perfil farmacológico e sociodemográfico foi determinado através da avaliação dos prontuários farmacoterapêuticos cedidos pela equipe multidisciplinar da UBS.

O Medication Regimen Complexity Index foi desenvolvido por George et al., e foi adaptado e validado para o português do Brasil em 2007 por Melchiors, 
Correr e Fernandéz-Llimós, sendo denominado de Índice da Complexidade da Farmacoterapia (ICFT). O ICFT é uma ferramenta dividida em três seções (A, B e C), sendo cada seção pontuada de acordo com as informações sobre o regime terapêutico dos pacientes. A parte A trata dos dados sobre formas de dosagens, a parte $\mathrm{B}$ faz referência às informações de frequências de doses e a parte $\mathrm{C}$ estabelece às informações adicionais para utilização dos medicamentos. O valor total do índice é calculado somando os pontos das três seções. Para quantificação do ICFT foi considerado o tratamento medicamentoso de uso contínuo dos pacientes, incluindo antidiabéticos e fármacos usados para outras condições patológicas, como hipertensão e dislipidemia $[7,9]$.

Para avaliação do perfil bioquímico e pressórico foi considerada a média aritmética da glicemia capilar, creatinina, pressão arterial sistólica (PAS) e diastólica (PAD) dos pacientes. Com o intuito de estimar o grau de funcionamento dos rins dos pacientes, a função renal ou glomerular, baseada na depuração de creatinina, foi determinada pela fórmula de Cockcroft-Gault:

$$
\text { Taxa de filtração glomerular (TFG) }=\frac{(140 \text {-idade }) \text { x peso }(\mathrm{kg})}{(\text { creatinina sérica } \times 72)}
$$

A unidade da TFG é expressa $\mathrm{mL} / \mathrm{min}$. Para mulheres, multiplica-se o valor da TFG por 0,85 [1]. De acordo com os valores da TFG, a doença renal do DM pode ser classificada da seguinte maneira: estágio 1 (TFG normal): $\geq 90 \mathrm{~mL} / \mathrm{min}$; estágio 2 (TFG levemente reduzida): 60 a $89 \mathrm{~mL} / \mathrm{min}$; estágio 3A (moderada redução): 45 a $59 \mathrm{~mL} / \mathrm{min}$; estágio 3B (redução marcada): 30 a 44mL/min; estágio 4 (redução grave): 15 a $29 \mathrm{~mL} / \mathrm{min}$; estágio 5 (insuficiência renal): $<15 \mathrm{~mL} / \mathrm{min} / 1,73 \mathrm{~m}^{2}[1]$.

Todos os dados foram tabulados no software Epi Info versão 3.5.2, e analisados no programa estatístico IBM SPSS Statistics 20. Efetuou-se análise descritiva das variáveis numéricas, sendo expressas como média e erro padrão da média. Para as variáveis nominais e ordinais foram utilizadas frequências relativas (\%) e absolutas (n). Para a análise inferencial recorreu-se à análise de correlação de Spearman (rho), após empregar teste de Shapiro-Wilk para verificar a normalidade das distribuições. Os seguintes valores, conforme Hulley et al., foram adotados para interpretar a força das correlações: coeficientes menores que 0,4 (fraca magnitude), entre 0,4 e 0,5 (moderada magnitude) e $>0,5$ (forte magnitude). O nível de significância de $5 \%(\mathrm{p}<0,05)$ foi adotado como rejeição da hipótese de nulidade [10].

\section{RESULTADOS}

A unidade básica de saúde do estudo apresentava um total de 79 prontuários de pacientes diabéticos. Destes, quatro apresentavam informações incompletas e foram excluídos. Dessa forma a amostra total deste estudo foi constituída por 75 indivíduos, 55 (73,3\%) eram do gênero feminino e 20 (26,7\%) do masculino. A média de idade foi de $59,1 \pm 1,8$ anos, com mínima de 13 e máxima de 91 anos, sendo que 52\% tinham 60 anos ou mais. Em relação aos medicamentos prescritos, os 75 participantes utilizavam 218 fármacos ao todo,

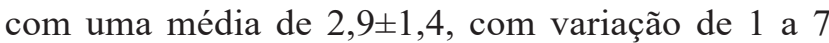
medicamentos para cada paciente (Tabela 1).

Em relação ao ICFT, foi encontrado uma média de $9,42 \pm 0,48$ pontos, com variação de 3 a 20,5. Em comparação com a seção A (formas de dosagens) e C (informações adicionais para utilização dos medicamentos), a seção $B$, relacionada às informações sobre as frequências de doses, apresentou a maior média. Os valores do ICFT foram maiores entre as mulheres (Figura 1).

Com relação às variáveis laboratoriais, verificou-se um valor médio da glicemia capilar $(n=64)$ de $217,5 \pm 9,10 \mathrm{mg} / \mathrm{dl}$, com variação de 116,33 a 385,33 $\mathrm{mg} / \mathrm{dl}$. A associação de metformina com glibenclamida estava presente na maioria dos pacientes, cujo valores glicêmicos atingiram a média de $195,32 \pm 11,54 \mathrm{mg} /$ dl (variação de 116,33 a $384 \mathrm{mg} / \mathrm{dl}$ ). No entanto, os participantes que utilizavam apenas um antidiabético oral, para o controle da glicemia, possuíam menores índices (Tabela 2).

O ICFT apresentou correlação direta e positiva de forte magnitude, estatisticamente significativa, com a glicemia capilar (Figura 2) $(\mathrm{p}<0,05)$. Pacientes com baixas pontuações (menor que 8 ) do ICFT $(n=29)$ apresentaram média de glicemia de $177,22 \pm 10,55 \mathrm{mg} / \mathrm{dl}$, com variação de 116,3 a $302 \mathrm{mg} / \mathrm{dl}$; já aqueles com as mais altas pontuações (maior que 15) do ICFT $(n=11)$ apresentaram média de glicemia de 279,10 $\pm 20,48 \mathrm{mg}$ / dl, com variação de 172 a $385 \mathrm{mg} / \mathrm{dl}$. Todas as seções do ICFT mostraram correlações estatisticamente significativas positivas de forte magnitude com a glicemia capilar $(\mathrm{p}<0,01)$ (Tabela 3 ).

Em relação a pressão arterial dos pacientes $(n=51)$, a média permaneceu nos valores de $134,8 \pm 3,3 \mathrm{mmHg}$ para PAS e $80,7 \pm 1,6 \mathrm{mmHg}$ para $\mathrm{PAD}$, com variação de 97,5 a $200 \mathrm{mmHg}$ da PAS e 60 a $120 \mathrm{mmHg}$ da PAD. Foi observado que o ICFT apresentou uma correlação positiva com a PAS (Figura 2) $(\mathrm{p}<0,05)$. Pacientes com baixas pontuações (menor que 8 ) do ICFT $(\mathrm{n}=22)$ apresentaram PAS de $122,9 \pm 3,8 \mathrm{mmHg}$, 
com variação de 97,5 a $200 \mathrm{mmHg}$; já aqueles com as mais altas pontuações (maior que 15) do ICFT $(n=10)$ apresentaram PAS de $153,5 \pm 7,9 \mathrm{mg} / \mathrm{dl}$, com variação de 110 a 200mmHg. Todas as seções do ICFT mostraram correlações estatisticamente significativas positivas de forte magnitude com PAS $(\mathrm{p}<0,05)$ (Tabela 3). Não houve correlação significativa da PAD com o ICFT.

A creatinina $(n=24)$ e a TFG $(n=16)$ foram $0,87 \pm 0,1 \mathrm{mg} / \mathrm{dl}$ e $100,24 \pm 11,8 \mathrm{ml} / \mathrm{min}$, respectivamente, com variação de 0,3 a $3,4 \mathrm{mg} / \mathrm{dl}$ da creatinina e 24,87 a $191,2 \mathrm{ml} / \mathrm{min}$ da TFG. A creatinina apresentou uma correlação direta e negativa, de fraca magnitude ao
ICFT (Figura 2) $(p<0,05)$. Já a filtração glomerular correlacionou-se de forma direta ao aumento do ICFT (Figura 2) $(\mathrm{p}<0,01)$. A média final do ICFT dos diabéticos com filtração glomerular menor que $90 \mathrm{ml} / \mathrm{min}(\mathrm{n}=5)$ foi de $9,0 \pm 1,0 \mathrm{ml} / \mathrm{min}$ e dos pacientes com filtração glomerular maior ou igual a $90 \mathrm{ml} / \mathrm{min}$ $(\mathrm{n}=11)$ foi de $12,1 \pm 1,4 \mathrm{ml} / \mathrm{min}$.

A seção B do ICFT e o valor médio da creatinina e da filtração glomerular apresentaram uma correlação estatisticamente significativas ( $p<0,05)$, com correlação total negativa de moderada magnitude e positiva de forte magnitude, respectivamente (Tabela 3 ).

Tabela 1. Grupos, subgrupos terapêuticos e princípios ativos dos medicamentos prescritos a pacientes com diabetes mellitus tipo 2, atendidos em uma unidade básica de saúde de Timon-MA, (n=218), e sua distribuição, segundo classificação Anatomical Therapeutic Chemical Classification System.

\begin{tabular}{|c|c|c|c|c|}
\hline & \multirow{2}{*}{ Medicamento } & \multirow{2}{*}{ Código ATC } & \multicolumn{2}{|c|}{ Frequência } \\
\hline & & & $\mathbf{n}$ & $\%$ \\
\hline \multicolumn{3}{|l|}{ Trato alimentar e metabolismo } & 132 & 60,7 \\
\hline \multirow[t]{5}{*}{ Medicamentos usados no diabetes } & Metformina & A10BA02 & 59 & 27,1 \\
\hline & Glibenclamida & А10ВB01 & 52 & 23,9 \\
\hline & Insulina NPH & A10AC01 & 18 & 8,3 \\
\hline & Vildagliptina & $\mathrm{A} 10 \mathrm{BH} 02$ & 1 & 0,5 \\
\hline & Glicazida & A10BB09 & 2 & 0,9 \\
\hline \multicolumn{3}{|l|}{ Sistema cardiovascular } & 79 & 36,4 \\
\hline \multirow[t]{3}{*}{ Agentes com ação sobre o sistema renina-angiotensina } & Losartana & C09CA01 & 18 & 8,3 \\
\hline & Enalapril & C09AA02 & 18 & 8,3 \\
\hline & Captopril & C09AA01 & 10 & 4,6 \\
\hline \multirow[t]{2}{*}{ Betabloqueadores } & Atenolol & C07AB03 & 7 & 3,2 \\
\hline & Propanolol & C07AA05 & 2 & 0,9 \\
\hline \multirow[t]{2}{*}{ Hipolipemiantes } & Sinvastatina & C10AA01 & 3 & 1,4 \\
\hline & Rosuvastatina & C10AA07 & 1 & 0,5 \\
\hline Diuréticos & Hidroclorotiazida & С03АA03 & 17 & 7,8 \\
\hline Bloqueadores dos canais de cálcio & Anlodipino & C08CA01 & 3 & 1,4 \\
\hline \multicolumn{3}{|l|}{ Sistema nervoso } & 2 & 1 \\
\hline \multirow[t]{2}{*}{ Psicoanalépticos } & Amitriptilina & N06AA09 & 1 & 0,5 \\
\hline & Citalopram & N06AB04 & 1 & 0,5 \\
\hline \multicolumn{2}{|l|}{ Sangue e órgãos hematopoiéticos } & & 5 & 2,3 \\
\hline Agentes antitrombóticos & Ácido acetilsalicílico & B01AC06 & 5 & 2,3 \\
\hline \multicolumn{3}{|l|}{ Total } & 218 & 100 \\
\hline
\end{tabular}

ATC: Anatomical Therapeutic Chemical Classification System.

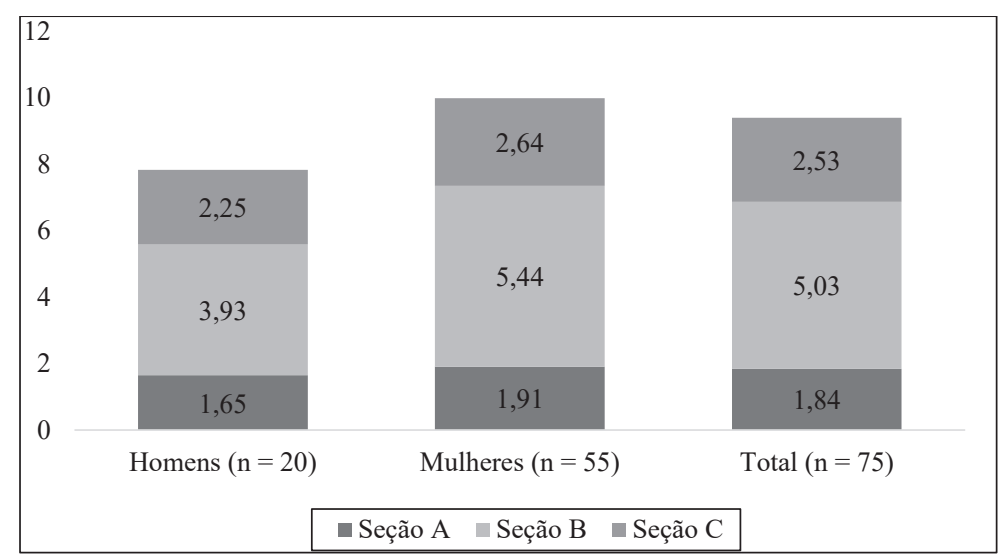

Figura 1. Média das pontuações do Índice de Complexidade da Farmacoterapia (ICFT), conforme as seções (A, B e C), de pacientes com diabetes mellitus tipo 2 atendidos em uma unidade básica de saúde de Timon-MA $(n=75)$. 
Tabela 2. Terapêutica farmacológica de pacientes com diabetes mellitus tipo 2 atendidos em uma unidade básica de saúde de Timon-MA e seus respectivos valores glicêmicos médios.

\begin{tabular}{lccc}
\hline Terapêutica farmacológica & $\begin{array}{c}\text { Glicemia média }(\mathbf{m g} / \mathbf{d l}) \\
\mathbf{\pm} \text { erro padrão da média }\end{array}$ & $\mathbf{n}$ & Frequência \\
\hline Metformina e Glibenclamida & $195,32 \pm 11,54$ & 33 & 51,56 \\
Insulina NPH & $267,33 \pm 13,26$ & 8 & 12,5 \\
Metformina & $180,04 \pm 22,87$ & 6 & 9,37 \\
Glibenclamida & $146,63 \pm 9,92$ & 4 & 6,25 \\
Metformina e Insulina NPH & $269,59 \pm 25,45$ & 7 & 10,94 \\
Metformina, Glibenclamida e Insulina NPH & $327,84 \pm 52,16$ & 2 & 3,13 \\
Outras & $281,50 \pm 28,38$ & 4 & 6,25 \\
Total & & 64 & 100 \\
\hline
\end{tabular}
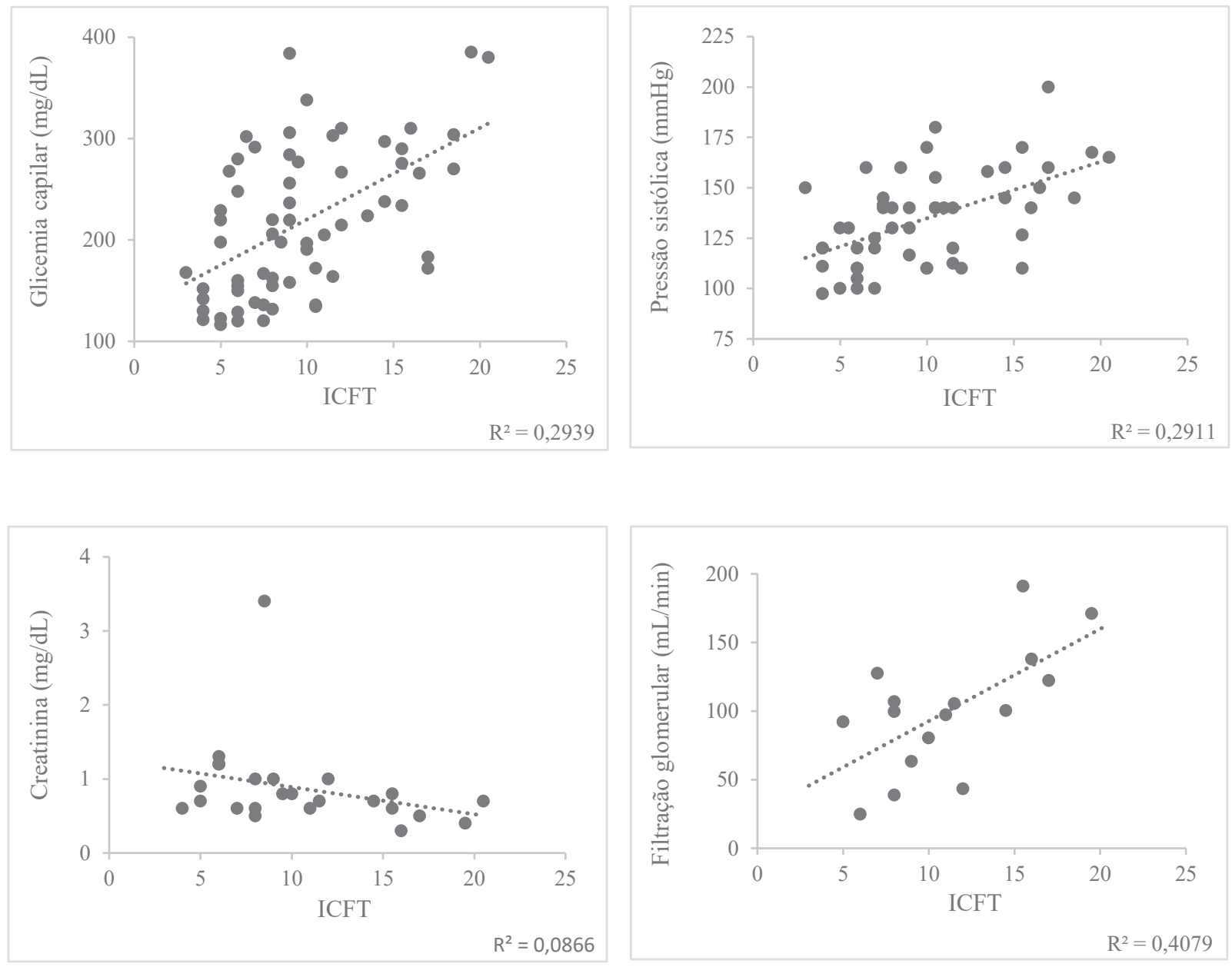

Figura 2. Correlação do Índice de Complexidade da Farmacoterapia (ICFT) e as variáveis do perfil bioquímico e pressórico de pacientes com diabetes mellitus tipo 2 atendidos em uma unidade básica de saúde de Timon-MA: I - Correlação do ICFT com glicemia capilar (mg/dL): $\mathrm{n}=64$; II - Correlação do ICFT com pressão sistólica $(\mathrm{mmHg}): \mathrm{n}=51$; III - Correlação do ICFT com creatinina (mg/dL): $\mathrm{n}=24$; IV - Correlação do ICFT com filtração glomerular (mL/min): $\mathrm{n}=16$. 
Tabela 3. Correlação, conforme magnitude, do índice de complexidade da farmacoterapia (ICFT) e as variáveis do perfil bioquímico e pressórico de pacientes com diabetes mellitus tipo 2 atendidos em uma unidade básica de saúde de Timon-MA.

\begin{tabular}{|c|c|c|c|c|}
\hline \multirow{2}{*}{ Variáveis } & \multicolumn{4}{|c|}{ ICFT } \\
\hline & Seção A & Seção B & Seção C & Total \\
\hline Glicemia capilar $(n=64)$ & 0,494 & 0,329 & 0,578 & 0,538 \\
\hline Magnitude & Moderada & Fraca & Forte & Forte \\
\hline Valor de $\mathrm{p}$ & $<0,001$ & 0,008 & $<0,001$ & $<0,001$ \\
\hline Pressão arterial sistólica $(n=51)$ & 0,295 & 0,570 & 0,330 & 0,520 \\
\hline Magnitude & Fraca & Forte & Fraca & Forte \\
\hline Valor de $\mathrm{p}$ & 0,035 & $<0,001$ & 0,018 & $<0,001$ \\
\hline Creatinina $(n=24)$ & $-0,279$ & $-0,413$ & $-0,308$ & $-0,406$ \\
\hline Magnitude & Fraca & Moderada & Fraca & Moderada \\
\hline Valor de $\mathrm{p}$ & 0,186 & 0,045 & 0,143 & 0,049 \\
\hline Filtração glomerular ( $n=16)$ & 0,441 & 0,542 & 0,381 & 0,566 \\
\hline Magnitude & Moderada & Forte & Fraca & Forte \\
\hline Valor de $\mathrm{p}$ & 0,087 & 0,030 & 0,146 & 0,022 \\
\hline
\end{tabular}

ICFT: Índice de Complexidade da Farmacoterapia.

\section{DISCUSSÃO}

Entre os pacientes diabéticos selecionados, nota-se predominância do sexo feminino em todas as faixas etárias. Dados semelhantes foram encontrados em outros estudos na atenção primária [11-13]. Quanto à idade, a predominância de pessoas diabéticas na faixa etária acima de 60 anos foi observada em outros estudos já realizados em UBS $[14,15]$.

A média de medicamentos (2,9 medicamentos para cada paciente) detectada em nosso estudo foi maior que as médias verificadas em outros estados como em Goiás $(2,4)$ e Sergipe $(2,8)[16,17]$, o que nos levou a inferir uma falta de acompanhamento clínico a esses indivíduos, uma vez que o excesso de medicamentos pode dificultar no tratamento dos indivíduos [18]. Em relação aos medicamentos utilizados, os principais antidiabéticos utilizados pelos participantes do estudo foram insulina, metformina e glibenclamida, ou seja, medicamentos que constam na Relação Nacional de Medicamentos Essenciais [19]. Observou-se uma total predominância da metformina e glibenclamida (Tabela 1), semelhante ao encontrado em outros estudos [20,21]. A metformina apresenta vantagem considerável em relação aos outros antidiabéticos orais, pois além de auxiliar no controle glicêmico, peso corporal, dispilidemia e pressão arterial diastólica, reduz eventos macrovasculares associados ao DM [22].

Em relação ao ICFT, a média foi de 9,42 pontos, muito aquém de outros estudos [23]. Esse valor inferior pode ser justificado principalmente pela ausência de informações nos prontuários dos pacientes, visto que o instrumento foi preenchido exclusivamente pelo pesquisador e não pelos profissionais da UBS, o que poderia ter tornado os resultados mais amplos. Quanto às seções do ICFT, Libby et al. destacaram a seção $\mathrm{B}$, frequência de doses, como a principal responsável pelo alto ICFT no DM, o que corrobora com o presente estudo, o que indica o ponto primordial de ação para simplificação dos esquemas farmacoterapêuticos [24].

Os valores glicêmicos dos pacientes apresentaram uma certa limitação, pois a indefinição do método utilizado na UBS (glicemia pós-prandial ou glicemia de jejum) ao realizar a glicemia capilar, dificultou o estabelecimento de valores limites para o diagnóstico dos pacientes diabéticos. Nesta perspectiva, o critério usado para definir o estado clínico dos pacientes do estudo, foi a glicemia casual, que segundo a Sociedade Brasileira de Diabetes seria aquela realizada a qualquer hora do dia, sem levar em consideração o intervalo da última refeição. Dessa forma, o valor médio obtido nos indivíduos $217,5 \mathrm{mg} / \mathrm{dL}$ superou o valor limite $\geq 200 \mathrm{mg} / \mathrm{dL}$, indicando um descontrole glicêmico [1].

Os diabéticos necessitam incluir em sua farmacoterapia inúmeros medicamentos com diferentes mecanismos de ação devido a progressão da doença requerer a inclusão de novos fármacos para atingir o controle glicêmico. Por conseguinte, era esperado que indivíduos com maior complexidade da farmacoterapia estivessem com um melhor manejo da glicemia. Entretanto, notou-se uma correlação proporcional, 
quanto mais elevado o nível da glicemia capilar, mais alto os valores do ICFT, sugerindo uma baixa adesão ao tratamento nos pacientes do estudo [25]. Paula e Souza encontraram resultados semelhantes, ressaltando que a utilização de uma quantidade maior de medicamentos antidiabéticos relaciona-se com um pior controle da glicemia [26].

Dos fatores que podem afetar a adesão ao tratamento, talvez o mais comum e o mais negligenciado seja a compreensão dos pacientes ao regime de tratamento, em que os médicos muitas vezes superestimam, o que acaba afetando, principalmente, as informações adicionais necessárias para ingerir ou administrar os medicamentos da farmacoterapia dos pacientes (seção C do ICFT). Nesta perspectiva, a seção C apresentou a maior influência no descontrole glicêmico $(\mathrm{rho}=0,583)$, visto que a maioria dos prontuários dos pacientes que utilizavam metformina e glibenclamida apresentavam orientações sobre o horário específico (manhã, tarde, noite) para ingerir o medicamento [27]. Estes resultados demonstraram a importância da orientação dos profissionais de saúde, ao instruírem toda a gestão da medicação, no processo de cuidado dos pacientes antes da ingestão dos medicamentos [28].

Em relação à pressão arterial, observou-se elevação discreta na PAS $(134,83 \mathrm{mmHg})$ e valores próximos ao normal da PAD $(80,68 \mathrm{mmHg})$, uma vez que os valores da pressão arterial a serem atingidas em indivíduos adultos devem ser $130 / 80 \mathrm{mmHg}$. O controle rígido da pressão arterial diminui consideravelmente o risco de progressão para as complicações crônicas vasculares do DM [1]. A contribuição dos componentes do ICFT (seções A, B e C) variou entre o regime terapêutico dos anti-hipertensivos e esquemas dos antidiabéticos. A forte correlação da seção B no descompasso da PAS indica uma maior frequência de doses dos medicamentos anti-hipertensivos, com uma menor quantidade de informações relacionadas à forma de consumo (seção C). Gupta et al. demonstraram que o uso de anti-hipertensivos combinados em uma única dose diária esteve associado a uma melhora significativa na aderência e persistência da terapia, e com possíveis tendências benéficas sobre os níveis da PA, em vez da combinação livre da medicação ingerida em diferentes momentos. Esses dados sugerem que a frequência de dosagem (seção B do ICFT) exerce um maior impacto na aderência e persistência do que a quantidade de dose do fármaco por comprimido (seção A do ICFT) [29].

Martínez et al. demonstraram que a complexidade da farmacoterapia elevada associou-se de forma direta ao agravamento da função renal, justificada pela elevada prescrição de medicamentos e esquemas posológicos aos indivíduos [25]. Contudo, os pacientes do presente estudo apresentam níveis normais de creatinina e da taxa de filtração glomerular, ou seja, um alto ou baixo valor do ICFT não prejudica de forma significativa a função renal.

A correlação inversamente proporcional da creatinina e a correlação diretamente proporcional da taxa de filtração glomerular com o ICFT, como observado na Figura 2, pode ser justificada, provavelmente, pelo efeito protetor renal dos medicamentos antihipertensivos e antidiabéticos. Os inibidores da enzima conversora de angiotensina causam uma elevação do fluxo sanguíneo renal ao provocar vasodilatação das arteríolas eferentes, atrasando a deterioração da taxa de filtração glomerular [30]. A metformina exerce sua ação reduzindo a resistência insulínica, que é um fator fundamental na progressão da doença renal crônica [31].

Segundo, Khan et al. a associação de múltiplos medicamentos: anti-hipertensivo, antidiabético e antilipêmico exerce maior redução nos valores de creatinina, e consequentemente aumento na taxa de filtração glomerular, que a administração isolada desses fármacos, isto justifica a correlação unicamente da seção B do ICFT com esses parâmetros, atribuindo a responsabilidade à frequência de doses dos medicamentos dos pacientes [32].

É importante ressaltar que o presente estudo se baseou em dados preexistentes, já registrados nos prontuários, apresentando, com isso, algumas limitações que não dependeram do controle do pesquisador. No entanto, as informações encontradas foram suficientes para apresentar resultados relevantes e confiáveis.

A partir dos resultados encontrados pôde-se constatar uma elevada complexidade da farmacoterapia, sendo a frequência de dosagem a principal responsável por este resultado. A complexidade do regime de medicamentos esteve associada a diversas variáveis, o que sugere uma forte influência da polifarmácia na adesão ao tratamento em diabéticos, provocando, principalmente, o agravamento do controle glicêmico e pressórico. Desta forma, conclui-se que o desenvolvimento de intervenções estratégicas para otimizar o regime medicamentoso e, consequentemente, melhorar a adesão ao tratamento é fundamental para melhora do controle glicêmico na diabetes.

\section{NOTAS}

\section{Apoio financeiro}

O presente trabalho foi realizado com apoio da Coordenação de Aperfeiçoamento de Pessoal de Nível Superior - Brasil (CAPES). 
Declaração de conflito de interesses

Os autores declaram não haver conflitos de interesses relevantes ao conteúdo deste estudo.

\section{Contribuições dos autores}

Todos os autores fizeram contribuições substanciais para concepção, ou delineamento, ou aquisição, ou análise ou interpretação de dados; e redação do trabalho ou revisão crítica; e aprovação final da versão para publicação.
Disponibilidade dos dados e responsabilidade pelos resultados

Todos os autores declaram ter tido total acesso aos dados obtidos e assumem completa responsabilidade pela integridade destes resultados.

\section{AGRADECIMENTOS}

Ao Centro Catarinense de Reabilitação, à Ullis Técnica Ortopédica e à Associação Cristã de Deficientes Físicos de Passo Fundo.

\section{REFERÊNCIAS}

1. Sociedade Brasileira de Diabetes. Diretrizes da Sociedade Brasileira de Diabetes (2015-2016). São Paulo: A.C. Farmacêutica; 2016.

2. Becker NB, Heleno MGV. A eficácia adaptativa em pessoas com diabetes mellitus tipo 2. Bol Psicol. 2016;66(145):159-70.

3. Gusso G, Lopes JMC. Tratado de medicina de família e comunidade. 2. ed. Porto Alegre: Artmed; 2018.

4. Carvalho CJ, Marins JCB, Amorim PRS, Fernandes MF, Reis HHT, Sales SS, Miranda MR, Lima LM. Altas taxas de sedentarismo e fatores de risco cardiovascular em pacientes com hipertensão arterial resistente. Medicina (Ribeirão Preto). 2016;49(2):124-33. https://doi.org/10.11606/issn.2176-7262.v49i2p124-133

5. Boas LCGV, Foss-Freitas MC, Pace AE. Adesão de pessoas com diabetes mellitus tipo 2 ao tratamento medicamentoso. Rev Bras Enferm. 2014;67(2):268-73. https://doi.org/10.11606/d.22.2009.tde-18082009-125600

6. Cramer JA. A systemic review of adherence with medications for diabetes. Diabetes Care. 2004;27(5):1218-24.

7. George J, Phun YT, Bailey MJ, Kong DC, Stewart K. Development and validation of the medication regimen complexity index. Ann Pharmacother. 2004;38(9):1369-76. https://doi.org/10.1345/aph.1d479

8. Favaro DTL, Santos MLSG, Sasaki NSGMS, Santos LHR, Vendramini SHF, Pompeo DA. Grupos educativos para o controle de hipertensão e diabetes mellitus: revisão integrativa de literatura. Arq Ciênc Saúde. 2017;24(1):7-14. https://doi.org/10.17696/2318-3691.24.1.2017.534

9. Melchiors AC, Correr CJ, Fernández-Llimos F. Tradução e validação para o português do Medication Regimen Complexity Index. Arq Bras Cardiol. 2007;89(4):210-18. https://doi.org/10.1590/s0066-782x2007001600001

10. Hulley SB, Cummings SR, Browner WS, Grady DG, Newman TB, Duncan MS. Delineando a pesquisa clínica: uma abordagem epidemiológica. 4. ed. Porto Alegre: Artmed; 2015.

11. Tavares DMS, Santos EA, Dias FA, Ferreira PCS, Oliveira PB. Fatores associados à qualidade de vida dos idosos com diabetes mellitus. Rev Enferm UFPE on line. 2014;8(6):1491-501.

12. Vicente NG, Goulart BF, Iwamoto HH, Rodrigues LR. Prevalence of adherence to the medical treatment of people with Diabetes Mellitus. Enferm. Glob. 2018;52:474-86.

13. Smaniotto M, Stakonski DS, Mick L, Teston L, Sbeghen MR. Aspectos epidemiológicos de pacientes com diabetes mellitus em uma unidade básica de saúde na cidade de Chapecó- SC. Biosaúde. 2015;17(1):13-20. https://doi. org/10.11606/d.25.2013.tde-14082013-093259

14. Winkelmann ER, Fontela PC. Condições de saúde de pacientes com diabetes mellitus tipo 2 cadastrados na Estratégia Saúde da Família, em Ijuí, Rio Grande do Sul, 2010-2013. Epidemiol Serv Saúde. 2014;23(4):665-74. https://doi. org/10.5123/s1679-49742014000400008

15. Barros JMS, Porto MLS. Perfil dos usuários diabéticos da unidade básica de saúde do município de Santana do Seridó-RN. Temas em Saúde. 2016;16(2):226-38. https://doi.org/10.12820/rbafs.23e0037

16. Borges LM, Silva EV. Análise dos indicadores de prescrição médica em uma unidade de saúde de Anápolis-GO. Rev Tempus Actas de Saúde Colet. 2010;4(3):63-72. https://doi.org/10.22533/at.ed.2681918023

17. Faraoni AS. Possíveis interações medicamentosas entre usuários de uma unidade básica de saúde (UBS) do município de São Cristóvão-SE. Rev Saúde Com. 2015;11(1):10-9. https://doi.org/10.11606/d.100.2014.tde-23012015-145117 
18. Cuentro V, Modesto T, Andrade MA, Silva MVS. Prevalência e fatores associados à polifarmácia entre idosos de um hospital público. Rev Contexto Saúde. 2016;16(30):28-35 https://doi.org/10.21527/2176-7114.2016.30.28-35

19. Brasil. Ministério da Saúde. Secretaria de Ciência, Tecnologia e Insumos Estratégicos. Departamento de Assistência Farmacêutica e Insumos Estratégicos. Relação nacional de medicamentos essenciais: Rename. Brasília: Ministério da Saúde, 2015. https://doi.org/10.1590/0103-1104201711217

20. Oliveira RC, Scalone FM, Lopes ACBA, Cabrera MAS. Analysis of the use of drugs in cardiovascular and antidiabetic primary health care according to age. Acta Sci Health Sci. 2013;35(1):119-23. https://doi.org/10.4025/ actascihealthsci.v35i1.10694

21. Barreto MNSC, Cesse EAP, Lima RF, Marinho MGS, Specht YS, Carvalho EMF, Fontbonne A. Análise do acesso ao tratamento medicamentoso para hipertensão e diabetes na Estratégia de Saúde da Família no Estado de Pernambuco, Brasil. Rev Bras Epidemiol. 2015;18(2):413-24. https://doi.org/10.11606/d.17.2017.tde-06012017-155647

22. Brasil. Ministério da Saúde. Secretaria de Atenção à Saúde. Departamento de Atenção Básica. Estratégias para o cuidado da pessoa com doença crônica: diabetes mellitus. Brasília: Ministério da Saúde; 2013. https://doi. org/10.11606/d.17.2019.tde-09112018-161800

23. Zanetti MOB, Moraes JL, Marchetti JM, Andrade RCG. Description of the complexity of prescribed medication regimens in primary health care of Ribeirão Preto-SP. Clin Biomed Res. 2018;38(1):1-7. https://doi.org/10.4322/23579730.76622

24. Libby AM, Fish DN, Hosokawa PW, Linnebur SA, Metz KR, Nair KV, Saseen JJ, Vande Griend JP, Vu SP, Hirsch JD. Patient-level medication regimen complexity across populations with chronic disease. Clin Ther. 2013;35(4):385-98. https://doi.org/10.1016/j.clinthera.2013.02.019

25. Martínez BB, Ferreira NC. Avaliação da complexidade da farmacoterapia em diabéticos. Rev Med Minas Gerais. 2012;22(2):133-8.

26. Paula e Souza RA, Correr CJ, Melchiors AC, Sanches ACC, Wiens A, Pontarolo R. Determinants of glycemic control and quality of life in type 2 diabetic patients. Lat Am J Pharm 2011;30(5):860-7

27. Pinto IVL, Reis AMM, Almeida-Brasil CC, Silveira MR, Lima MG, Ceccato MGB. Avaliação da compreensão da farmacoterapia entre idosos atendidos na Atenção Primária à Saúde de Belo Horizonte, MG, Brasil. Ciênc Saúde Colet. 2016;21(11):3469-81. https://doi.org/10.1590/1413-812320152111.19812015

28. Brune MFSS, Ferreira EE, Ferrari CKB. O Método Dáder na atenção farmacêutica em pacientes hipertensos no município de Pontal do Araguaia-MT, Brasil. Mundo Saúde 2014;38(4):402-9. https://doi.org/10.15343/01047809.20143804402409

29. Gupta AK, Arshad S, Poulter NR. Compliance, safety, and effectiveness of fixed-dose combinations of antihypertensive agents: a meta-analysis. Hypertension. 2015;55(2):399-407. https://doi.org/10.1161/ hypertensionaha.109.139816

30. Texeira JRS. Tratamento farmacológico da hipertensão em diabéticos: estudo comparativo: experiência profissionalizante na vertente de Farmácia Comunitária e Investigação [dissertação]. Covilhã: Universidade da Beira Interior; 2013. https://doi.org/10.22355/exaequo.2017.36.10

31. Almeida FA. O uso de metformina em pacientes diabéticos com insuficiência renal. É tempo de mudança. Rev Fac Ciênc Méd Sorocaba. 2015;17(1):1-14.

32. Khan RA, Siddiq A, Baig SG. Assessment of renal parameters following combined administration of antihypertensive, hypolipidemic and hypoglycemic drugs. Am J Drug Deliv. 2015;3(1):79-85. 